\title{
Peri-operative Chemotherapy in Patients with Oesophageal and Gastro-oesophageal Junction Cancer - Three Years of Experience
}

\section{Pechačová Z. ${ }^{1}$, Zemanová M. ${ }^{1}$, Haruštiak T. ${ }^{2}$, Vítek P. ${ }^{3}$, Fencl P. ${ }^{4}$}

1Department of Oncology, First Faculty of Medicine, Charles University in Prague and General University Hospital in Prague, Prague, Czech Republic;

${ }^{2} 3^{\text {rd }}$ Department of Surgery, First Faculty of Medicine, Charles University in Prague and University Hospital Motol, Prague, Czech Republic;

${ }^{3}$ Institute of Radiation Oncology, First Faculty of Medicine, Charles University in Prague and Hospital Bulovka, Prague, Czech Republic;

${ }^{4}$ PET Centre, Hospital Na Homolce, Prague, Czech Republic

Received November 6, 2012; Accepted April 8, 2013.

Key words: Oesophageal and gastro-oesophageal junction adenocarcinoma Peri-operative chemotherapy

Abstract: Peri-operative chemotherapy has been found to benefit patients with oesophageal and gastro-oesophageal junction adenocarcinoma. This study's aim was to evaluate the efficacy and tolerance of this treatment. The study included patients with carcinoma of the lower oesophagus and gastro-oesophageal junction in whom the disease was evaluated as potentially operable. Chemotherapy (CHT) consisted of three preoperative and three postoperative cycles of intravenous epirubicin and cisplatin on day 1 plus a continuous infusion of fluorouracil for 21 days (ECF) or oral capecitabine for 14 days (ECCap). Postoperative radio-chemotherapy (CRT) with fluorouracil or capecitabine after $\mathrm{CHT}$ was indicated in patients with two and more positive lymph nodes. Sixty-three patients started the treatment. Median follow-up was 32 months. Preoperative CHT was completed by 62 patients,

This study was supported by a grant from the Ministry of Health of the Czech Republic IGA MZ CR NT12331-5/2011.

Mailing Address: Milada Zemanová, MD., PhD., Department of Oncology, First Faculty of Medicine, Charles University in Prague and General University Hospital in Prague, U nemocnice 2, 12808 Prague 2, Czech Republic; Phone: +420 224962 219; e-mail: milada.zemanova@vfn.cz 
52 had surgery, 46 had radical resection, 25 patients had pN0 and 21 patient pN plus findings. Postoperative $\mathrm{CHT}$ was started in $39(62 \%)$ patients and completed in $32(51 \%)$. Ten (16\%) patients had postoperative CRT. Adverse events of grade 3 and 4 were: neutropenia $17 \%$, vomiting $8 \%$, fatigue $5 \%$, diarrhoea $3 \%$. Reasons for omitting surgery in $11(17 \%)$ patients were: progression in 7 patients, medically unfit in 3 patients, other in 1 patient. In the reporting period there were recurrences in 39 of all patients, in 7 locoregional only, in 10 distant plus locoregional, and in 19 distant metastases. Median survival was 24.1 months and 3 -year survival rate was $42 \%$. Peri-operative chemotherapy ECF/ECCap was feasible and well tolerated. Radical resection was performed in most patients.

\section{Introduction}

Approximately 500 new cases of oesophageal carcinoma occur in the Czech Republic each year, with mortality greater than $90 \%$ (Institute of Health Information and Statistics of the Czech Republic, 2011). The incidence of adenocarcinomas of the lower thoracic oesophagus and gastro-oesophageal junction (GEJ) has been increasing very rapidly in recent years and proportion of adenocarcinomas among oesophageal cancers in 2009 had reached 40\% (Institute of Health Information and Statistics of the Czech Republic, 2011, unpublished data from the Czech cancer registry). In the absence of distant metastases, surgery is the primary treatment modality and enables 5 -year survival of approximately $20 \%$ (lyer et al., 2004). The addition of adjuvant or neoadjuvant therapy is widely accepted apart from in stage T1N0 (Enestvedt et al., 2010). An optimal treatment approach has not been established. Antineoplastic treatment could be delivered as preoperative concomitant radio-chemotherapy (CRT) (Walsh et al., 1996; Reynolds et al., 2007), which, according to a multivariate analysis from 2007, improves survival by $13 \%$ over 2 years (Gebski et al., 2007). However, the preoperative CRT increases the operative morbidity and mortality to approximately $10 \%$ (Fiorica et al., 2004). According to the multivariate analysis (Gebski et al., 2007), preoperative chemotherapy $(\mathrm{CHT})$ brings similar benefit by improving survival in adenocarcinomas as does concurrent CRT (Medical Research Council Oesophageal Cancer Working Group, 2002; Boige et al., 2007). Adenocarcinomas of the lower oesophagus and GEJ have for several years been included into clinical trials together with gastric cancers. Postoperative concomitant CRT (Macdonald et al., 2001) in the INT-0116 study demonstrated improved survival of patients after resection of the gastric carcinoma, GEJ and lower oesophagus when lymph nodes were involved or when the level of local risk was defined to be in stages Ib-IV. Survival was improved to 35 vs. 27 months after 6-year follow-up, and thus postoperative CRT is one option for standard treatment of GEJ cancers.

Finally, in patients with gastric and GEJ cancers, peri-operative $\mathrm{CHT}$ demonstrated benefit in the randomized study known as MAGIC (Cunningham 
et al., 2006). In this study, patients with potentially resectable gastric (74\%) or GEJ and distal oesophagus $(26 \%)$ cancers were randomized to undergo either surgery alone or peri-operative $\mathrm{CHT}$ ( 3 cycles preoperative and 3 cycles postoperative) consisting of cisplatin, epirubicin and 5 -fluorouracil. The group of patients treated with $\mathrm{CHT}$ showed longer survival, at $36 \%$ vs. $23 \%$ after 5 years. The treatment was well tolerated and postoperative morbidity and mortality were similar in both arms of the study. Peri-operative CHT was therefore accepted as a standard treatment approach in GEJ cancers.

The significance of radiotherapy in the multimodal treatment protocol has not been proven definitively, as a comparison between preoperative CHT and CRT showed no significant difference (Stahl et al., 2009) and a comparison between postoperative CRT and CHT had never yet been published as of the time of presenting our study findings. Nevertheless, postoperative CRT seems to be a logical option, and especially in patients with lymph nodes positivity despite preoperative treatment and who have a poorer prognosis (Reynolds et al., 2007).

The modified MAGIC protocol of peri-operative $\mathrm{CHT}$ with the application of postoperative (chemo)radiotherapy in high-risk patients having two and more affected lymph nodes has been established at the Department of Oncology, General University Hospital in Prague in cooperation with the $3^{\text {rd }}$ Department of Surgery, University Hospital Motol and Institute of Radiation Oncology, Hospital Bulovka (all of which departments are parts of the First Faculty of Medicine, Charles University in Prague).

The aim of this study was to evaluate the efficacy and tolerability of multimodal treatment in patients with carcinoma of the distal oesophagus and GEJ.

\section{Material and Methods}

\section{Eligibility}

Previously untreated patients of any age with carcinoma of the lower oesophagus or GEJ according to the Siewert classification (Siewert and Stein, 1996), in operable stage, excluding T1N0-1M0, were indicated for the treatment with perioperative CHT. The patients must have had World Health Organization (WHO) performance status 0 or 1 and functional status determined by spiroergometry and echocardiography suitable for a two-cavity surgical approach (thoracotomy plus laparotomy), without uncontrolled cardiac disease or creatinine clearance below $60 \mathrm{ml}$ per minute. Patients must have had proven histology of adenocarcinoma, or exceptionally squamous carcinoma, present in the GEJ. Indication was approved by a multidisciplinary team. The staging was specified according to UICC 6 and evaluated by computed tomography (CT) or positron emission tomography - computed tomography (18FDG-PET-CT), endosonography and esophagogastroscopy. In cases involving juxtaregional lymph nodes (stage IV), the patients enrolled in this evaluation were evaluated by the surgeon in advance as resectable. 


\section{Chemotherapy and radiotherapy}

The treatment was designed as three preoperative and three postoperative cycles of $\mathrm{CHT}$, and the surgery had to be performed 3-6 weeks after completing the third cycle. Chemotherapy consisted of epirubicin $\left(50 \mathrm{mg} / \mathrm{m}^{2}\right)$ and cisplatin $\left(60 \mathrm{mg} / \mathrm{m}^{2}\right)$ intravenously with hydration and standard antiemetic prophylaxis on day 1 plus a continuous infusion of fluorouracil $\left(200 \mathrm{mg} / \mathrm{m}^{2}\right.$ per day) with portable infusion pump for 21 days (ECF) or oral capecitabine $\left(1,000 \mathrm{mg} / \mathrm{m}^{2}\right.$ twice a day) for 14 days (ECCap) in a 21 days cycle. In all patients, left ventricular ejection fraction (EF) was measured by echocardiography before $\mathrm{CHT}$ start and epirubicin was omitted in patients with history of ischemic heart disease or ejection fraction below $50 \%$. Before each cycle of $\mathrm{CHT}$, a complete blood count, serum creatinine, electrolytes and liver function were determined. Dose modification was recommended for cisplatin if there was a rise in serum creatinine level and for fluoropyrimidines if non-haematological toxicity of grade 2 and higher was evidenced in the previous cycle by such symptoms as stomatitis, hand-foot syndrome or diarrhoea. In case of myelosuppression on day 22 (neutropenia less then $1.5 \times 10^{9} / /$ or thrombocytopenia less than $\left.100 \times 10^{9} / I\right)$, the cycle was postponed until blood count had returned to normal values.

Postoperative CRT was indicated in patients with two and more positive lymph nodes in a surgical specimen and was included sequentially after the completion of postoperative $\mathrm{CHT}$.

Total dose of 50 Gy in 25 fractions, 5 fractions/week, was prescribed. Clinical target volume (CTV) included gastric remnant, anastomosis plus $3-4 \mathrm{~cm}$ cranial margin and lymph node regions as follows (regardless if subjected to lymph node dissection or not): inferior para-oesophageal (lower mediastinal), perigastric and coeliac. Gamma radiation $18 \mathrm{MeV}$ was delivered, employing technologies of intensity-modulated radiation therapy (IMRT) and image-guided radiation therapy (IGRT). If a dose distribution was acceptable, a less-demanding 3D conformal radiation therapy (3DCRT) was employed. Concomitant $\mathrm{CHT}$ was prescribed, either fluorouracil continuous infusion $\left(200 \mathrm{mg} / \mathrm{m}^{2} /\right.$ day $)$ or capecitabine $\left(625 \mathrm{mg} / \mathrm{m}^{2} /\right.$ day), both on radiotherapy days (i.e. weekend off).

\section{Surgery}

After preoperative $\mathrm{CHT}$, re-examination by endoscopy was performed as well as CT scan or 18FDG-PET/CT scan of the chest and upper abdomen. Resectability was assessed and the operation followed 3-6 weeks after CHT unless it was contra-indicated or refused by the patient. Patients with resectable disease underwent either transthoracic oesophagectomy with gastric pull-up reconstruction with intrathoracic oesophago-gastric anastomosis (Ivor Lewis procedure) or proximally extended total or proximal gastrectomy depending on location and extent of tumour. All resected patients had intraoperatively proven negative proximal resection margin by frozen-section histopathology 
analysis. Abdominal D2 lymphadenectomy was a part of all radical operations, and infracarinal 2-field lymphadenectomy was added to transthoracic oesophagectomy. Dissection of cervical lymph nodes was selectively performed in patients with preoperative clinical suspicion of cervical nodal involvement based on 18FDG-PET/CT examination.

\section{Statistical methods}

All statistical analyses were performed in Statistica, version 7.0 (StatSoft, 2004; www.statsoft.com). Mean values were computed as well as standard deviations to describe joint parameters. Survival curves were constructed according to the

Table 1 - Characteristics of the patients $(\mathrm{N}=63)$

\begin{tabular}{|c|c|}
\hline \multicolumn{2}{|l|}{ Sex - no. (\%) } \\
\hline Men & 57 (90\%) \\
\hline Women & $6(10 \%)$ \\
\hline \multicolumn{2}{|c|}{ Age (years) - range, median 37-74, median 59} \\
\hline \multicolumn{2}{|l|}{ Histology - no. (\%) } \\
\hline adenocarcinoma & 60 (95\%) \\
\hline squamous carcinoma & $3(5 \%)$ \\
\hline \multicolumn{2}{|c|}{ TN stage (UICC 6) - no. (\%) } \\
\hline T2No & $2(3 \%)$ \\
\hline $\mathrm{T} 2 \mathrm{~N} 1$ & $3(5 \%)$ \\
\hline T3N0 & $15(24 \%)$ \\
\hline T3N1 & $34(54 \%)$ \\
\hline T4N0 & $5(8 \%)$ \\
\hline T4N1 & $4(6 \%)$ \\
\hline \multicolumn{2}{|l|}{ Stage (UICC 6) - no. (\%) } \\
\hline 0,1 & $0(0 \%)$ \\
\hline$\| \mathrm{A}$ & $16(25 \%)$ \\
\hline IIB & $2(3 \%)$ \\
\hline III & $38(60 \%)$ \\
\hline IVA & $3(5 \%)$ \\
\hline IVB & $4(6 \%)$ \\
\hline \multicolumn{2}{|c|}{ Siewert classification - no. (\%) } \\
\hline distal oesophagus & $3(5 \%)$ \\
\hline GEJ 1 & $25(40 \%)$ \\
\hline GEJ 2 & $18(29 \%)$ \\
\hline GEJ 3 & $11(17 \%)$ \\
\hline GEJ without specification & $6(10 \%)$ \\
\hline
\end{tabular}


Kaplan-Meier method and their differences by log rank test. The Cox proportional hazards model was applied for both univariate and multivariate analyses of risk factors for survival and time to progression. All data were evaluated at significance level $\alpha=5 \%$ and all statistical tests used were bilateral. Normality of values distribution was verified prior to performing parametric statistical tests.

\section{Results}

Characteristics of the patients

Sixty-three patients started the treatment according to this protocol with curative intent in the time period from 1 March 2008 to 16 June 2010. The follow-up in the evaluated group of patients was at least 18 months. Characteristics of the patients are presented in Table 1. For staging evaluation, the TNM classification version UICC 6 was used. Stage T3 was most common, occurring in 49 patients (78\%), with $34(54 \%)$ cases of suspected lymph nodes (T3N1) and 15 (24\%) cases without (T3N0). Clinical N1 disease was found in $65 \%$ of cases. If Siewert classification (Siewert and Stein, 1996) was used, we recognized most tumours as being localized above or in the junction line - Siewert 1 (40\%), Siewert 2 (29\%) or distal oesophagus (5\%). All three patients with squamous cancer included in this study had tumour in the GEJ area (Siewert 1 in two and Siewert 2 in one patient).

\section{Chemotherapy}

Preoperative CHT was started in 63 and completed in 62 (98\%) patients (in 1 case the treatment strategy was changed after the first cycle to palliative CRT due to clinical signs of progression). Postoperative CHT was started in

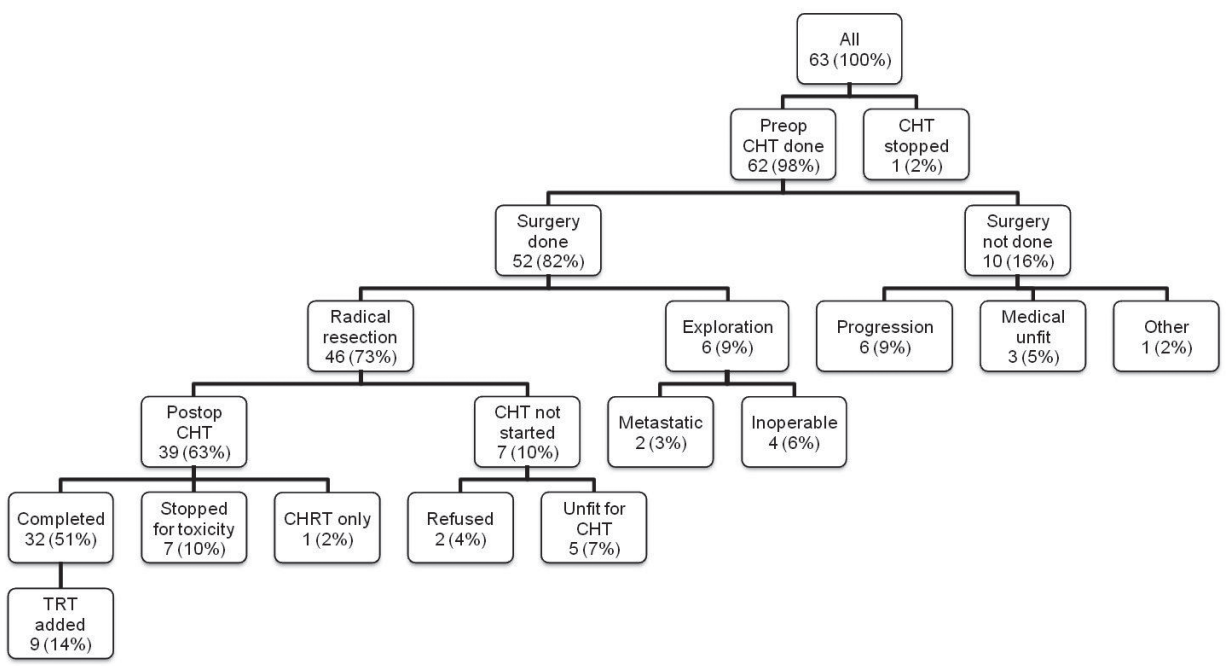

Figure 1 - Flow diagram.

CHT - chemotherapy; CHRT - chemoradiotherapy; TRT - teleradiotherapy 
39 (62\% of all) patients and was completed in 32 (51\% of all) patients. The ECF schedule was administered in 44 cases $(70 \%)$ and the ECCap schedule in 14 cases (22\%). Other schedules were chosen in 5 cases (8\%): once cisplatin and epirubicin were replaced with docetaxel due to excessive emetogenicity; in 1 patient $\mathrm{CHT}$ without epirubicin was used; in 1 case cisplatin was replaced with carboplatin because of neurotoxicity; and in 1 case docetaxel was used in monotherapy. One patient received only adjuvant chemoradiotherapy, and in an additional 9 cases sequential CRT or radiation therapy after postoperative CHT was done. Six patients did not complete the postoperative treatment due to intolerance. Flow diagram of treatment see on Figure 1.

\section{Surgery}

Out of 52 operated patients (82\%), 6 were found to have unresectable disease due to preoperatively unrecognized distant metastases (2 patients) or locally unresectable tumour (4 patients). Forty-six patients ( $73 \%$ of all, $88.5 \%$ of those operated) had the radical resection (i.e. the R0 resection rate was $88.5 \%$ ). Surgical finding pNO was found in 25 patients (54\% of R0 resections), with 3 cases of complete pathological response in this number (e.g. $6.5 \%$ of $\mathrm{R} 0$ resections). $\mathrm{pN}$ plus was found in 21 cases (46\% of R0).

Reasons for omitting surgery in $11(18 \%)$ patients were: local progression in 2 cases, distant metastases in 5 cases, medical unfitness in 3 patients, other reasons in 1 patient. A stent was implanted because of oesophageal obstruction in 7 patients with locally unresectable finding. No death was reported postoperatively within a period of 30 days.

\section{Radiotherapy}

The radiotherapy was part of postoperative therapy "per treatment protocol" in 10 cases. In 5 other cases the radiotherapy was indicated "off protocol" as local therapy because of the locally unresectable surgical finding in 2 cases or when surgery had not been done in 3 cases. Eleven patients underwent the radiotherapy at the Institute of Radiation Oncology, First Faculty of Medicine, Charles University in Prague and Hospital Bulovka, and the other patients in other hospitals according to their places of residence. The concomitant $\mathrm{CHT}$ was administered in 9 cases $(60 \%)$. In 2 cases, the radiotherapy was discontinued before the appointed time because of intolerance.

\section{Chemotherapy side effects and treatment modification}

Most adverse events during the administration of $\mathrm{CHT}$ were neutropenia, fatigue, weakness and gastrointestinal intolerance (Table 2).

The $\mathrm{CHT}$ had to be modified for various reasons, which are summarized in Table 3 . We needed to postpone $\mathrm{CHT}$ in 24 patients (38\%) - during preoperative CHT in 19 cases (30\%) and during postoperative treatment in 13 cases (33\%). We had to 
Table 2 - Chemotherapy toxicity

\begin{tabular}{lcc}
\hline \multicolumn{1}{c}{ Type of the toxicity } & grade $1-4-$ no. (\%) & grade $3-4-$ no. (\%) \\
\hline Neutropenia & $32(51 \%)$ & $10(17 \%)$ \\
Febrile neutropenia & $1(2 \%)$ & $1(2 \%)$ \\
Fatigue and weakness & $33(52 \%)$ & $5(8 \%)$ \\
Nausea and vomiting & $33(52 \%)$ & $3(5 \%)$ \\
Diarrhoea & $16(25 \%)$ & $2(3 \%)$ \\
Obstipation & $16(25 \%)$ & $0(0 \%)$ \\
Anaemia & $15(24 \%)$ & $0(0 \%)$ \\
Weight loss & $12(19 \%)$ & $0(0 \%)$ \\
Stomatitis & $7(11 \%)$ & $0(0 \%)$ \\
Neurotoxicity & $7(11 \%)$ & $1(2 \%)$ \\
Thromboembolic disease & $6(10 \%)$ & $2(3 \%)$ \\
Nephrotoxicity & $5(8 \%)$ & $0(0 \%)$ \\
Psychical decompensation & $4(7 \%)$ & $0(0 \%)$ \\
Thrombocytopenia & $3(5 \%)$ & $0(0 \%)$ \\
Hand-foot syndrome & $2(3 \%)$ & $0(0 \%)$ \\
\hline
\end{tabular}

Table 3 - Chemotherapy modification due to toxicity

\begin{tabular}{lcc}
\hline \multicolumn{1}{c}{ Treatment modification, reason } & $\begin{array}{c}\text { Preoperative } \\
\text { chemotherapy }\end{array}$ & $\begin{array}{c}\text { Postoperative } \\
\text { chemotherapy }\end{array}$ \\
\hline No. $(\%)$ & $\mathrm{N}=63$ & $\mathrm{~N}=39$ \\
\hline Postponing of the cycle & $19(30 \%)$ & $13(33 \%)$ \\
\hline neutropenia & $14(22 \%)$ & $10(26 \%)$ \\
gastrointestinal intolerance & $2(3 \%)$ & $2(5 \%)$ \\
thromboembolic disease & $1(2 \%)$ & $1(3 \%)$ \\
other & $2(3 \%)$ & $2(5 \%)$ \\
\hline Reducing the dose & $4(6 \%)$ & $11(28 \%)$ \\
\hline neutropenia & $2(3 \%)$ & $6(15 \%)$ \\
stomatitis & $1(2 \%)$ & $0(0 \%)$ \\
hand-foot syndrome & $1(2 \%)$ & $1(3 \%)$ \\
general deterioration & $0(0 \%)$ & $3(8 \%)$ \\
other & $0(0 \%)$ & $2(5 \%)$ \\
\hline Termination before the appointed time & $1(2 \%)$ & $6(15 \%)$ \\
\hline diarrhoea & $1(2 \%)$ & $1(17 \%)$ \\
vomiting & $0(0 \%)$ & $4(67 \%)$ \\
thromboembolic disease & $0(0 \%)$ & $2(33 \%)$ \\
general deterioration & $1(2 \%)$ & $3(50 \%)$ \\
\hline
\end{tabular}


reduce the dose of the cytostatics in 11 patients (17\% of all) - during preoperative treatment in 4 cases $(6 \%)$ and during postoperative cycles in 11 cases $(28 \%$ of those with postoperative $\mathrm{CHT}$ ).

The $\mathrm{CHT}$ had to be discontinued before the appointed time during preoperative therapy in 1 patient because of diarrhoea and general deterioration. The postoperative $\mathrm{CHT}$ had to be discontinued in 6 patients (15\%) due to serious grade of toxicity.

After the surgery, 13 patients (25\%) were not indicated for postoperative CHT, 2 patients refused, 6 patients had progression, 4 patients had general deterioration, and 1 case had a diagnosis of a second malignancy.

\section{Survival and recurrences}

Median follow-up in the time of analysis was 32 months, with a range of 19-46 months. In the reporting period 34 (54\%) patients died. The median survival was 24.1 months while 1 -, 2 - and 3 -year survival were $70 \%, 50 \%$ and $42 \%$, respectively (Figure 2). The median time to progression was 13.3 months. Evaluation of different variables (age, gender, stage at diagnosis, $\mathrm{R} 0$ resection, postoperative pathology) in relation to survival probability showed that the most important difference was found in patients having negative lymph nodes in the resection specimen $(N=25)$ in comparison with lymph nodes positive resections $(\mathrm{N}=21)$, with their 3-year survival being $82 \%$ and $38 \%$ respectively (Figure 3 ).

In the reporting period there were confirmed recurrences in $39(62 \%)$ of all patients while $22(35 \%)$ remained recurrence-free. The situation was not known in 2 cases $(3 \%)$, but these patients died. Recurrence rate in radically resected patients

Kaplan-Meier survival curve

- Events $\bullet$ Censored

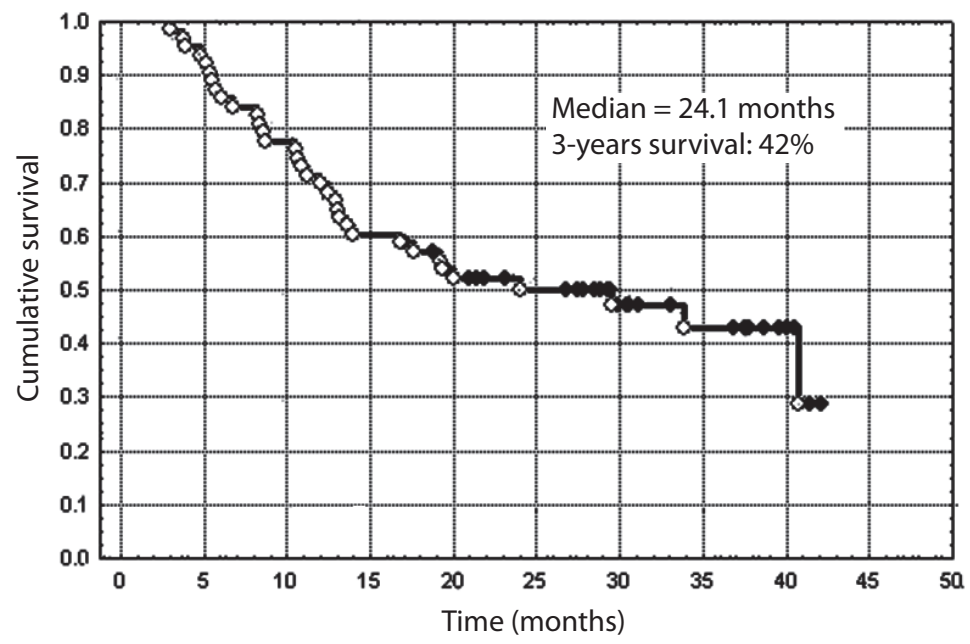

Figure 2 - Overall survival. 


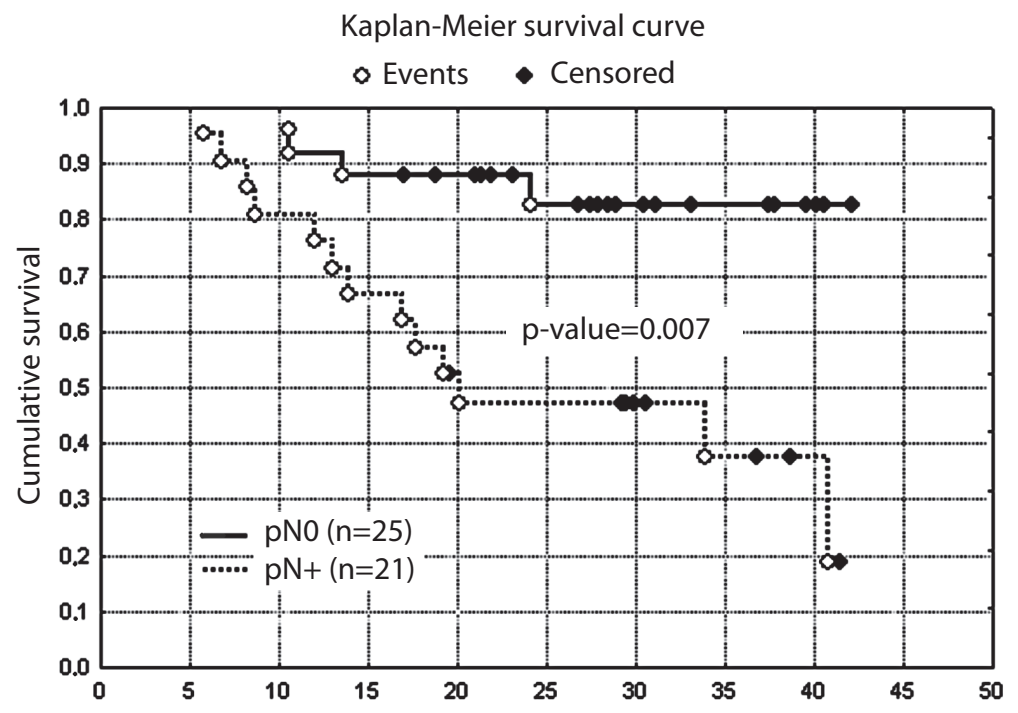

Figure 3 - Survival of different postoperative stages.

was 23 cases (50\%), 20 patients (43\%) were recurrence-free, and the situation for 2 (4\%) was not known. In patients with pN0, 7 from 25 (28\%) recurred. In the case of pN1, 16 from 21 (76\%) recurred. The most frequent cause of recurrence was distant metastases in 33 (85\%) patients - alone in 24 (62\%) patients and in $10(26 \%)$ cases distant plus locoregional concurrently (for the locations see Table 4). Locoregional recurrence developed in 13 patients (33\%), and in $5(13 \%)$ patients the recurrence was locoregional alone.

The recurrence rate in relation to pathologic stage at surgery is summarized in Table 5, and from these numbers the fact is obvious that the recurrence rate correlates with advanced surgical finding and that the time to recurrence also is shorter with advanced surgical finding.

\section{Discussion}

This study showed 3-year results from managing patients suffering with adenocarcinoma of the GEJ and distal oesophagus. The therapy consisted of peri-operative chemotherapy, resection and chemoradiotherapy added in high-risk cases. The preoperative course of chemotherapy was completed by $98 \%$ of our patients and postoperative chemotherapy by $51 \%$, which compares favourably with the original MAGIC study (Cunningham et al., 2006) having $86 \%$ completing preoperative and $42 \%$ postoperative chemotherapy. Surgery was performed in $82 \%$ and radical resection in $73 \%$ of all patients, but the $\mathrm{R} 0$ resection rate (comparison of RO and all resection) was $88.5 \%$. Here, too, comparison with the MAGIC study (Cunningham et al., 2006) is relevant because here among 229 operated patients the "curative" resection was achieved in 169 of randomized patients (i.e. 
the $\mathrm{R} 0$ resection rate was $74 \%$ ). Our results also can be seen as similar to those recently published from a German phase II study (Thuss-Patience et al., 2012) on peri-operative chemotherapy using docetaxel plus cisplatin plus capecitabine in 51 patients with gastro-oesophageal adenocarcinoma. In that study, $94.1 \%$ of patients received all three planned cycles preoperatively and $52.9 \%$ received three cycles postoperatively. The $\mathrm{R} 0$ resection rate was $90.2 \%$, and $13.7 \%$ of patients showed complete pathological remission (PCR). PCR in our set was found in $5 \%$ of patients. Evidence that the preoperative chemotherapy was well tolerated

\section{Table 4 - Recurrences localization}

\begin{tabular}{lc}
\hline Recurrence location & No. (\%) \\
\hline $\begin{array}{l}\text { Total } \\
\text { Locoregional }\end{array}$ & $39(100 \%)$ \\
\hline Total & $13(33 \%)$ \\
Only locoregional & $5(13 \%)$ \\
\hline Distant metastases & \\
Total & $33(85 \%)$ \\
Only distant & $24(62 \%)$ \\
lymph nodes & $10(33 \%)$ \\
lung & $9(30 \%)$ \\
abdominal cavity & $8(27 \%)$ \\
bones & $5(16 \%)$ \\
brain & $3(10 \%)$ \\
liver & $3(10 \%)$ \\
muscles & $2(10 \%)$ \\
more distant locations & $10(33 \%)$ \\
\hline Distant plus locoregional concurrently & $10(26 \%)$ \\
\hline
\end{tabular}

Table 5 - Recurrences rate relative to pathologic stage at surgery

\begin{tabular}{lccc}
\hline Pathologic stage at surgery & $\begin{array}{c}\text { Patients } \\
\text { no. }\end{array}$ & $\begin{array}{c}\text { Recurrences } \\
\text { no. }\end{array}$ & $\begin{array}{c}\text { Recurrences rate } \\
\% \text { in the group }\end{array}$ \\
\hline All patients & 63 & 41 & $65 \%$ \\
Total operated & 52 & 30 & $60 \%$ \\
R0 resections & 46 & 23 & $50 \%$ \\
PCR & 3 & 1 & $33 \%$ \\
PT1-4N0 & 22 & 6 & $27 \%$ \\
PN0 (including PCR) & 25 & 7 & $28 \%$ \\
PTanyN+ & 21 & 16 & $76 \%$ \\
PM1 & 2 & 2 & $100 \%$ \\
exploratory surgery & 4 & 4 & $100 \%$ \\
without surgery & 11 & 11 & $100 \%$ \\
\hline
\end{tabular}


and of the good quality of surgery was provided can be seen in the fact that no postoperative death was reported in the period of 30 days.

We used schedule ECF more often than ECCap in our group of patients because capecitabine was not accessible until 2009 for this indication. The schedule with capecitabine - (ECCap) - was given preference toward the end of the period, as it was better tolerated, lower-cost and easily served. Regimens with capecitabine have been preferred for similar reasons also in other studies published recently (Okines et al., 2010; Thuss-Patience et al., 2012).

In analysing the toxicity profile of $\mathrm{CHT}$ in separate cycles, we can recognize the good tolerability of preoperative CHT. Higher grades of toxicity (3-4) occurred in less than $5 \%$ of patients, except that neutropenia was detected in $17 \%$ (but only 1 case of febrile neutropenia set in). The tolerance of postoperative CHT was somewhat poorer, with a notably higher frequency of gastrointestinal and general symptoms; similarly as in the MAGIC study (Cunningham et al., 2006).

The median and maximal time of follow-up in our group was a bit shorter than in the MAGIC study (Cunningham et al., 2006). We thus had no data about 5-year survival, but 3 -year survival of $42 \%$ in our study and the death rate at the time of analysis (54\% in our set versus $55 \%$ in MAGIC) was similar.

Our study reported only patients with tumours in the distal oesophagus or GEJ, and in similar numbers as were the subsets of the 65 patients (37 lower oesophagus, 28 oesophago-gastric junction) in the MAGIC study (Cunningham et al., 2006). We had a different distribution of sub-localisations due to using Siewert classification (Siewert and Stein, 1996) as to the location of oesophago-gastric junction tumours. Nodal status pN0 in $54 \%$ of patients with $\mathrm{R} 0$ resection in our set could be considered an indicator of chemotherapy efficacy, being about $20 \%$ in a set of patients with surgery only (Walsh et al., 1996). The patients with pN0 status had significantly longer survival compared using univariate analysis to pN+ (Figure 3). Nodal status pN0 has been reported to be a positive prognostic factor in several studies (Reynolds et al., 2007; Zemanova et al., 2010).

According to the multivariate analysis (Gebski et al., 2007), preoperative CHT had similar significant benefit for improving survival in adenocarcinomas as did concurrent CRT. The single randomized study comparing effect of preoperative CHT or CRT showed no significant difference (Stahl et al., 2009), but it did show a positive survival trend ( $47 \%$ vs. $28 \%$ in 3 -year survival) in favour of chemoradiotherapy as well as higher complete pathological response rate (15.6\% vs. $2.0 \%)$. Postoperative mortality was non-significantly higher also in the CRT arm (10.2\% vs. 3.8\%). In our study, higher risk of postoperative death and other postoperative complications after neoadjuvant chemoradiotherapy was the sole reason for omitting this modality. On the other hand, radiotherapy does seem to be important for clearing of micrometastases in regional lymph nodes. It seems logical to use radiotherapy postoperatively (Macdonald et al., 2001) with more systemic treatment to get better eradication of distant micrometastases. There 
are few randomized data in this setting. The ARTIST trial (Lee et al., 2012) was the first study to our knowledge to investigate the role of postoperative CRT in patients with curatively resected gastric cancer and D2 lymph node dissection. This trial was designed to compare cytostatic treatment with capecitabine plus cisplatin $(\mathrm{XP})$ versus $\mathrm{XP}$ plus radiotherapy with capecitabine (XP/XRT/XP). In the subgroup of patients with pathologic lymph node metastasis at the time of surgery $(n=396)$, patients randomly assigned to the XP/XRT/XP arm experienced superior diseasefree survival when compared with those who received $X P$ alone $(P=0.0365)$, and the statistical significance was maintained in multivariate analysis (estimated hazard ratio, 0.6865; 95\% Cl, 0.4735-0.9952; P=0.0471). Presently, enrolment into the "CRITICS" study is ongoing. This is designed as preoperative chemotherapy in all patients and postoperative chemotherapy or chemoradiotherapy (Dikken et al., 2011). The contribution of postoperative radiation in our set of patients is difficult to evaluate because of the small number of subjects treated. Moreover, not all of our nodespositive patients had postoperative radiation; some of them had early recurrence, poor performance status or refused the treatment. This factor could be important, because surgically positive lymph nodes were clearly a negative prognostic factor for survival (Figure 3) by univariate analysis. In our next studies, we must continue to identify characteristics of unique, clinically informative patient subgroups - such as by using HER2 overexpression in GEJ cancers (24-35\%) (Gravalos and Jimeno, 2008) or diffuse histology - that may serve as the basis for more effective, tailored therapeutic approaches in patients with gastric adenocarcinoma, and optimally in a preoperative setting. Evidently, so-called non-responders, representing $40-55 \%$ of all patients undergoing neoadjuvant therapy, benefit little or not at all from it. Some data are available to indicate that it is possible to use the metabolic-anatomic information from 18FDG-PET/CT examinations for early identification of patients with good response to neoadjuvant chemotherapy for adenocarcinoma of the oesophagus and EGJ early after the first cycle (Langer et al., 2009). Confirming this approach, nonresponders could by treated alternatively - with immediate surgery or preoperative/ definitive chemoradiotherapy. Such strategy has been approved for study under project title "Early assessment of effectiveness of neoadjuvant chemotherapy for carcinoma of the oesophagus and oesophago-gastric junction using 18FDG-PET/CT", EudraCT number: 2011-001856-12, and the report from that study could provide the first findings as to the efficacy and feasibility of that protocol.

\section{Conclusion}

Peri-operative chemotherapy combining epirubicin, cisplatin and fluorouracil or capecitabine was feasible and well tolerated in our set. The patients having negative lymph nodes in the resection specimen $(\mathrm{N}=25)$ had better 3 -year survival in comparison with lymph nodes positive patients ( $N=21),(82 \%$ and $38 \%$ respectively). When surgery was omitted, it was mostly due to diagnosis of distant metastases - detected before or during the surgery. 


\section{References}

Boige, V., Pignon, J., Saint-Aubert, B., Lasser, P., Conroy, T., Bouché, O., Segol, P., Bedenne, L., Rougier, P., Ychou, M. (2007) Final results of a randomized trial comparing preoperative 5-fluorouracil (F)/cisplatin (P) to surgery alone in adenocarcinoma of stomach and lower esophagus (ASLE): FNLCC ACCORD07-FFCD 9703 trial. J. Clin. Oncol. 25, 4510.

Cunningham, D., Allum, W. H., Stenning, S. P., Thompson, J. N., van de Velde, C. J., Nicolson, M., Scarffe, J. H., Lofts, F. J., Falk, S. J., Iveson, T. J., Smith, D. B., Langley, R. E., Verma, M., Weeden, S., Chua, Y. J., MAGIC Trial Participants (2006) Perioperative chemotherapy versus surgery alone for resectable gastroesophageal cancer. N. Engl. J. Med. 355, 11-20.

Dikken, J. L., van Sandick, J. W., Maurits Swellengrebel, H. A., Lind, P. A., Putter, H., Jansen, E. P., Boot, H., van Grieken, N. C., van de Velde, C. J., Verheij, M., Cats, A. (2011) Neo-adjuvant chemotherapy followed by surgery and chemotherapy or by surgery and chemoradiotherapy for patients with resectable gastric cancer (CRITICS). BMC Cancer 11, 329.

Enestvedt, C. K., Perry, K. A., Kim, C., McConnell, P. W., Diggs, B. S., Vernon, A., O'Rourke, R. W., Luketich, J. D., Hunter, J. G., Jobe, B. A. (2010) Trends in the management of esophageal carcinoma based on provider volume: treatment practices of 618 esophageal surgeons. Dis. Esophagus 23(2), 136-144.

Fiorica, F., DiBona, D., Schepis, F., Licata, A., Shahied, L., Venturi, A., Falchi, A. M., Craxì, A., Cammà, C. (2004) Preoperative chemoradiotherapy for oesophageal cancer: a systematic review and meta-analysis. Gut 53(7), 925-930.

Gebski, V., Burmeister, B., Smithers, B. M., Foo, K., Zalcberg, J., Simes, J., Australasian Gastro-Intestinal Trials Group (2007) Survival benefits from neoadjuvant chemoradiotherapy or chemotherapy in oesophageal carcinoma: a meta-analysis. Lancet Oncol. 8(3), 226-234.

Gravalos, C., Jimeno, A. (2008) HER2 in gastric cancer: a new prognostic factor and a novel therapeutic target. Ann. Oncol. 19(9), 1523-1529.

Institute of Health Information and Statistics of the Czech Republic, National Oncology Register (2011) Cancer Incidence 2008 in the Czech Republic. Institute of Health Information and Statistics of the Czech Republic, Prague.

lyer, R., Wilkinson, N., Demmy, T., Javle, M. (2004) Controversies in the multimodality management of locally advanced esophageal cancer: Evidence-based review of surgery alone and combined-modality therapy. Ann. Surg. Oncol. 11, 665-673.

Langer, R., Ott, K., Feith, M., Lordick, F., Siewert, J. R., Becker, K. (2009) Prognostic significance of histopathological tumor regression after neoadjuvant chemotherapy in esophageal adenocarcinomas. Mod. Pathol. 22(12), 1555-1563.

Lee, J., Lim do, H., Kim, S., Park, S. H., Park, J. O., Park, Y. S., Lim, H. Y., Choi, M. G., Sohn, T. S., Noh, J. H., Bae, J. M., Ahn, Y. C., Sohn, I., Jung, S. H., Park, C. K., Kim, K. M., Kang, W. K. (2012) Phase III trial comparing capecitabine plus cisplatin versus capecitabine plus cisplatin with concurrent capecitabine radiotherapy in completely resected gastric cancer with D2 lymph node dissection: the ARTIST trial. J. Clin. Oncol. 30(3), 268-273.

Macdonald, J. S., Smalley, S. R., Benedetti, J., Hundahl, S. A., Estes, N. C., Stemmermann, G. N., Haller, D. G., Ajani, J. A., Gunderson, L. L., Jessup, J. M., Martenson, J. A. (2001) Chemoradiotherapy after surgery compared with surgery alone for adenocarcinoma of the stomach or gastroesophageal junction. N. Engl. J. Med. 345(10), 725-730.

Medical Research Council Oesophageal Cancer Working Group (2002) Surgical resection with or without preoperative chemotherapy in oesophageal cancer: a randomised controlled trial. Lancet 359, 1727-1734.

Okines, A. F., Ashley, S. E., Cunningham, D., Oates, J., Turner, A., Webb, J., Saffery, C., Chua, Y. J., Chau, I. (2010) Epirubicin, oxaliplatin, and capecitabine with or without panitumumab for advanced esophagogastric 
cancer: Dose-finding study for the prospective multicenter, randomized, phase II/III REAL-3 trial. Clin. Oncol. 28(25), 3945-3950.

Reynolds, J. V., Muldoon, C., Hollywood, D., Ravi, N., Rowley, S., O’Byrne, K., Kennedy, J., Murphy, T. J. (2007) Long-term outcomes following neoadjuvant chemoradiotherapy for esophageal cancer. Ann. Surg. 245(5), 707-716.

Siewert, J. R., Stein, H. J. (1996) Carcinoma of the cardia: Carcinoma of the gastroesophageal junction classification, pathology and extent of resection. Dis. Esophagus 9, 173-182.

Stahl, M., Walz, M. K., Stuschke, M., Lehmann, N., Meyer, H. J., Riera-Knorrenschild, J., Langer, P., EngenhartCabillic, R., Bitzer, M., Königsrainer, A., Budach, W., Wilke, H. (2009) Phase III comparison of preoperative chemotherapy compared with chemoradiotherapy in patients with locally advanced adenocarcinoma of the esophagogastric junction. J. Clin. Oncol. 27(6), 851-856.

Thuss-Patience, P. C., Hofheinz, R. D., Arnold, D., Florschütz, A., Daum, S., Kretzschmar, A., Mantovani-Löffler, L., Bichev, D., Breithaupt, K., Kneba, M., Schumacher, G., Glanemann, M., Schlattmann, P., Reichardt, P., Gahn, B. (2012) Perioperative chemotherapy with docetaxel, cisplatin and capecitabine (DCX) in gastrooesophageal adenocarcinoma: a phase II study of the Arbeitsgemeinschaft Internistische Onkologie (AIO). Ann. Oncol. 23(11), 2827-2834.

Walsh, T. N., Noonan, A., Hollywood, D., Kelly, A., Keeling, N., Hennessy, T. P. (1996) A comparison of multimodal therapy and surgery for esophageal adenocarcinoma. N. Engl. J. Med. 335(7), 462-467.

Zemanova, M., Petruzelka, L., Pazdro, A., Kralova, D., Smejkal, M., Pazdrova, G., Honova, H. (2010) Prospective non-randomized study of preoperative concurrent platinum plus 5-fluorouracil based chemoradiotherapy with or without paclitaxel in esophageal cancer patients: long-term follow-up. Dis. Esophagus 23(2), 160-167. 